Part II

THEORETICAL ASPECTS 


\title{
MAGNETOHYDRODYNAMICS OF AN ACTIVE REGION
}

\author{
H.U. SCHMIDT \\ (Max-Planck-Institut für Physik und Astrophysik, München, Germany)
}

\begin{abstract}
The dynamics of the magnetic fields which are imbedded into the non-stationary outer layers of the Sun show many facets of interest to observers and theoreticians alike. In a short review I can only deal with a small number of them and occasionally glance at some others. I hate to call these magnetic fields frozen into a matter which is rather in a boiling state, but the electrical conductivity in these layers is high enough to keep matter and magnetic flux together for rather long times, so that we can discuss the most important questions within the framework of magnetohydrodynamics with infinite conductivity. I will first talk mainly about the layers below the photosphere, where the matter controls the motion of the field, secondly about the intermediate state near the photosphere, where matter and field have comparable energy, and finally about the upper layers where the field controls the material motion.
\end{abstract}

\section{Dynamotheory of the Solar Cycle}

The cycle of solar activity ought to be explained as an oscillating dynamo with a period of 22 years. Some theories relevant to this problem have been put forward already many years ago by Cowling (1946), Elsasser (1950,1956), Bullard (1955), and Parker (1955). The most detailed model for a theory of the solar cycle was derived by Babcock (1961) from observations with the use of the earlier theories. It was developed further by Leighton (1964), and also by Kopecký (1966), and Godoli (1966). Let me briefly discuss some aspects of the Babcock model and some of its theoretical problems and implications. I will also occasionally touch upon other theories.

Babcock calls the solar cycle a free-running oscillator, lacking stabilization and driven by the differential rotation. Leighton visualizes it as a relaxation oscillation. The fast rotation near the solar equator stretches any meridional flux within a few years into an almost toroidal topology, in which the fieldlines trail behind their equatorial vertex. This implies a strong amplification of the field, which ends when a hypothetical twist instability or the magnetic buoyancy brings substantial parts of the flux to the solar surface therewith producing the active regions of a new cycle. The follower spot in a bipolar group appears usually at a higher latitude than the corresponding preceding spot and this tilt of about $6^{\circ}$ with respect to the equator is much larger than the average tilt of the almost toroidal field below the photosphere can be after years of differential rotation. The enhanced tilt may be due to Coriolis

Kiepenheuer (ed.), Structure and Development of Solar Active Regions, 95-107. O. I.U. 
forces, as was supposed by Cowling (1966). Whatever its reason, this tilt is essential for the dynamo action in this model. If the stretches of fieldlines above the surface are tilted in one direction, the stretches below the surface must become tilted the other way by the same token. In the rapid formation of active regions at the peak of the cycle this process will piecewise and locally turn over the subphotospheric field into a tilt which is against the grain of the differential rotation. When this is accomplished the latter will rotate the field further by almost $180^{\circ}$. The rotating field at first relaxes into a roughly polar field and then it is again wound up into an almost toroidal field opposite to the original one and ready for the second half-cycle. It is by no means clear whether the intermediate new polar field has connected fieldlines all the way from pole to pole or not.

This model is so closely connected to some basic facts of the solar cycle which are well established by observation that it hardly can be completely wrong. But besides the problems posed by the spectacular large scale features discussed this morning by Dr. Howard and Dr. Dodson-Prince, this model encounters some theoretical problems, which I want to comment on.

(1) Obviously the flux is very buoyant only during the phase of amplification. Babcock (1961) argues that the differential rotation acts like a roller bearing on the flux and forms flux ropes. Excessive local twisting then causes an instability which brings a stitch of this rope up into the surface to form a new active center. This proposal has two difficulties. The twisted field in the Northern hemisphere has a right-hand thread which should show up in all Northern spots. But this is not observed. Furthermore the rising stitch in the overtwisted rope must relax the twist and therefore rotate anticlockwise in the Northern hemisphere. This is inconsistent with the observed clockwise tilt of the axis between newly formed bipolar spots. Contrary to the observed tilt a strong twist would tend to preserve the original poloidal component and thereby counteract the solar cycle quite severely.

But if the roller-bearing mechanism is ruled out what causes the formation of a fairly uniform buoyant flux unit of some $10^{21}$ maxwell responsible for the average active region? This question was answered by Weiss (1964a, b, 1966). He shows that throughout the convection zone the convection must expel the flux from the cells. The amplified toroidal flux beneath the sunspot zone is concentrated into toroidal tubes of some $10^{21}$ maxwell. The flux will be compressed by the convection until the field intensity suppresses the convection inside the tube almost entirely, which affords about 5000 gauss in the deep convection zone. The buoyancy of these tubes is proved for all parts which have a radius of curvature larger than 0.17 of the solar radius. Near the surface the buoyant tubes must diverge to intensities of a few hundred gauss, since neither convective nor thermal energies can balance more.

(2) The tilt of the axis of an active region should be caused by Coriolis forces. In the sunspot zone the horizontal Coriolis force needs a day of unimpeded uniform motion to rotate the resulting displacement by $6^{\circ}$. Such motions can be found in a super- 
granulation cell which seems to last for 1 day (cf. Simon and Leighton, 1964). The same timescale holds for the appearance of a new active center. The circulation in a supergranule builds up the tilt of the axis between opposite base points of fieldlines in two steps. The upward motion in the center of the cell brings a stitch of the toroidal flux tube to the surface and subsequently the horizontal flow at the top of the cell moves the basepoints of the fieldlines apart toward the vertices of the cell. In both steps the rotation is clockwise in the Northern hemisphere and the total motion takes about 1 day.

(3) An oscillating dynamo can hardly operate without some kind of dissipation to dispose of the fields which are not in phase. But the usual dissipative processes which are due to particle interaction are probably not of primary importance in this context. The described dynamo should work for some periods even without these processes, i.e. for infinite conductivity because it can act on finite stretches of subphotospheric fieldlines in active latitudes just as well as on fieldlines extending all the way from pole to pole. It is rather the finite length of these stretches than the dissipation of flux which allows their permanent rotation. We may say that the rise of flux into the atmosphere provides the dissipative mechanism for the solar dynamo.

Of course the phases of activation of all the field stretches in the different latitudes would soon be put off their stroke if there occurs no reconnection between them. But solar cycles are certainly not completely in step for all latitudes, as Gnevyshev (1967) has just shown for the recent cycles. The many irregularities which solar activity exhibits, e.g. the so-called long-term cycles, or the very interesting fine-scale polarity changes in the polar caps as studied by Severny (1965), or even in moderate latitudes by Livingston (1967) are very encouraging for theoreticians because all these facts are evidence in favor of a long timescale for the final dissipation of solar magnetic flux crossing the photosphere. There is no reason why this timescale cannot be as long as some tens of years, just what it should be accounting for the diffusion and dissipation of flux by the surface convection. Diffusion timescales of this order were derived by Leighton (1964). If this concept is applicable, each solar latitude could act on its own for a timescale of years or so, and this seems to be in good agreement with observational findings.

(4) The flux crossing the surface is subject to the granular and supergranular convective motions. Leighton (1964) has shown that they act like a two-dimensional diffusivity on the footpoints of the fieldlines, at least if we consider scales large compared to a single cell. This random walk of the footpoints combined with their differential rotation causes the well-observed expansion, stretching and weakening of the aged bipolar magnetic regions, the cancellation of the polar cap field, and the equatorial leftovers called UM regions (cf. Bumba et al., 1966).

But the random walk also mixes fields of opposite polarity, so that they can be dissipated in small bits by local currents near to the photosphere where the conductivity has its minimum. In agreement with the local Cowling timescale this dissipation 
will reconnect the subphotospheric fields slowly but sufficiently to provide a weak coupling between the different latitudes and thereby a weak coherency of the phases in the solar cycle. The upper branches of the reconnected fieldlines will move into the corona and possibly into the solar wind.

(5) In Babcock's model solar activity takes its energy from the differential rotation, and this poses the problem where the latter gets its energy from. Kippenhahn (1963) has argued that this energy can be supplied from meridional circulations, which may be caused by anisotropic eddy viscosity in the convection zone. Plaskett $(1959,1962)$ and Weiss (1965) have discussed meridional differences of the superadiabatic gradient in the convection zone due to rotation as a possible driving mechanism for circulation and differential rotation. These authors argue that the solar magnetic field is too weak to interfere with the differential rotation, which otherwise should depend on the solar cycle.

(6) Another problem is posed by the amplitude of the oscillating magnetic activity which must be determined by a non-linear effect. The basic process which turns the tilt of the subphotospheric field over when part of the field rises above the surface would not contradict an ever-growing amplitude, since the newly formed reversed meridional flux can be produced from the strongly enhanced toroidal flux. The flux of the old cycle through the polar cap and the remnant UM regions are no match for this flux and they themselves must be proportional to the amplitude.

The most probable limiting mechanism is the following: The buoyancy of the fluxtubes must work against dragforces so that the upward drift becomes more rapid for the larger fluxtubes. Since the fluxtubes will grow by merger of neighboured tubes during the amplification, major activity at the surface will start, when the total intensity of subphotospheric flux has reached a certain critical level. Once this critical level is reached the further amplification of the meridional flux can be stopped by a reversal in the poloidal flux. Such a mechanism would be consistent with the fact that strong cycles have a short rise time.

(7) The most challenging problem for the Babcock model and for any dynamo theory of the solar cycle seems now to be posed by the recurrence tendency of active regions and by the simultaneous appearence of similar active centers at opposite longitudes which was demonstrated so perceptually by Dr. Dodson this morning. She pointed out that there is a systematic difference between the shifts in longitude for the individual active regions and for the location of recurrence itself. Obviously the individual active regions participate in the differential rotation, whereas the location of the recurrence does not. This difference points to very deep layers of the convection zone as for the cause of the recurrence. We may witness the structure of convection at the botton of the zone in the large dimensions, in the recurrence periods, and in the very long lifetimes of the system of recurrent centers, or 'giant granules' as Dr. Bumba calls them. But we also witness an ordered magnetic structure, probably a circular fluxtube, which seems to have a particularly unstable mode for two azimuthal nodes. This should have a simple explanation, but does not have it yet. 
Before I leave the subject I should mention a few other dynamo models. The stochastic dynamo action of the Coriolis forces in the solar convection zone was studied in detail by Steenbeck and Krause (1966) and Krause (1965), who developed a very promising linear theory for a purely oscillatory dynamo driven by the differential rotation. In this theory the necessary dissipation is assumed to be homogeneous throughout the convection zone which may need modification, since the most important dissipation is provided by the rise of magnetic flux into the atmosphere and by reconnection of fieldlines in the photosphere. Iroshnikov (1965) has put forward an investigation on the instability of a pregiven stationary subphotospheric field aligned in a surface of constant angular velocity. He finds instabilities in modes somewhat similar to the observed behaviour during the first half of a cycle, but of course for small amplitudes, so that no field reversal can be described. Finally I should mention in this context the work of Lortz (1967), who has constructed an exact stationary solution of the dynamo equations in helical symmetry, which fulfills reasonable boundary conditions.

\section{Formation of Plages and Spots}

What happens to the magnetic flux when its own buoyancy and the convection bring it up into the photosphere to form a new active region? Naturally it must arrive at this interface as a horizontal field stretching over a limited region for which upward motion prevails. We expect that the flux of an active region, usually some $10^{21}$ maxwell, will appear distributed over an area of a very few supergranules within a few lifetimes of the latter, i.e. within a few days.

The first phenomenon of a new active region is a plage and it has roughly the described features. Stepanov and Grigorev (1966) with the new Sibizmir-Magnetograph found transversal and longitudinal fields of about 200 gauss in bright plages, and Dr. Bappu presented similar results in his paper. These observations are consistent with an equipartition of energy between the photospheric convective motions and the magnetic field as estimated by Weiss (1964b) and De Jager (1964). They also imply that the flux of a sizable spot can be found in one supergranulation cell. Though any new flux elements or fieldlines should arrive first in the rising middle part of the convection elements, their basepoints will immediately be driven to the border of these elements, where the convection currents sink into the Sun and leave the flux behind. The observational evidence for this process is overwhelming for the supergranulation and to a lesser degree for the granulation, as is well known from the work of Simon and Leighton (1964), Bumba and Howard (1965), Sheeley (1966), and many others. Theoretical work on the expulsion of magnetic flux from eddies was done by Parker (1963a) and in detailed and thorough computations by Weiss (1964a, 1966), who presented part of his spectacular results in a most beautiful movy. He as well as Simon and Leighton (1964) pointed out that in nearly polygonal convection cells the flux becomes even more concentrated in the polygon vertices than simply at 
the borderlines of the cells. The fieldlines which connect these basepoints through the chromosphere and corona will therefore cover the cells with a nearly horizontal field, which must be responsible for the obviously aligned fine-structure in $\mathrm{H} \alpha$, which can be observed on high resolution filtergrams. It is also not surprising that Bumba and Howard (1965) found that the plages as well as the spots of an active region begin to appear just at the corners of supergranulation cells and probably in intergranular space. Bumba (1966) even found that active regions arise in the corners of still larger elements, which he calls 'giant granules'. It is interesting to note that the diameter of these structures of $400000 \mathrm{~km}$ corresponds nicely to the depth of the convection zone of about $100000 \mathrm{~km}$ if one applies the ratio for diameter and depth derived by Weiss (1966). But it is hard to imagine how so large an element can form an eddy.

The equipartition fields in the photosphere of about 200 gauss are not sufficient to reduce the convection in the deeper layers. But they are able to increase the exitation of sound and hydromagnetic waves near to the photosphere (cf. Kulsrud, 1955; Pikelner, 1960; Parker, 1964). In this way the enhancement of the non-radiative flux in an active region by an order of magnitude may be understood (cf. Livshitz, 1965).

The most obvious phenomenon in an active region is the sunspot. It is a local deficiency of temperature, radiative flux and pressure. The latter is balanced by the compressed magnetic field which may reach 5000 gauss. Various models by Deinzer (1965), Weiss (1964b), De Jager (1964), and Yun (1967) describe the observed spots quite well and explain the deficiency of radiative flow at the surface by a systematic reduction of the convective energy flow in the underlying convection zone. Modifying an early suggestion by Biermann (1941) they assume that such a reduction is caused by the magnetic field. According to these models the relative temperature or pressure deficiency becomes negligible at a depth of some $10^{3} \mathrm{~km}$, so that the spot is a rather shallow entity. The only thing which must persist below this depth is the magnetic flux which, rather than being disordered by the convection, will be compressed by it into a flux tube containing all the parallel flux of the active region, as Weiss (1964b) has suggested.

The energy flux which is missing in the radiative flux escaping from the sunspot must get out somehow and somewhere. Danielson (1965) argues that this flux, which amounts to about $\frac{1}{3}$ of the total flux over the sunspot area, can hardly be non-radiative flux into the local chromosphere overlying the spot, because the missing flux of one big spot would already account for $\frac{1}{6}$ of the non-radiative flux supplied into the whole chromosphere. Therefore most of this missing flux will probably be distributed over such a large area that it can escape as radiation from the photosphere without being detectable as a surplus. Whether this energy can be transported away from the spot by magnetohydrodynamic waves is an interesting problem which is intrinsically connected with the problem of the modes of convection in a strong magnetic field, as became clear from the work of Danielson $(1965,1966)$. But convection itself may already divert the energy flow sufficiently. 
The mechanism by which the missing flux is transported away from the magnetized upper layer of the convection zone underlying the umbra of a sunspot is rather efficient and selective, since the magnetic-field strength in an umbra falls into a narrow range between 1000 and 5000 gauss. As Zwaan (1965) has pointed out the cooling process seems to be sufficient for just this range but overly effective for intermediate field strengths, so that the field strength may flip flop between this range and the range below 200 gauss, which provides only a minor enhancement of the energy losses of the quiet photosphere (cf. Livshitz, 1965). Zwaan (1967) argues that the tiny magnetic regions with roughly $10^{3}$ gauss and diameters of about $1^{\prime \prime}$ found by Sheeley (1966, 1967) and Beckers and Schröter (1966) should be considered 'invisible sunspots' (cf. Hale and Nicholson, 1938) which are stabilized by the same mechanism but do not have an umbra due to the inflow of radiation above the layer at which the cooling mechanism is effective.

To summarize, it seems to be conceivable that sunspots are formed by the supergranulation which concentrates the flux in its vertices, where the magnetized matter at the top of the convection zone is cooled by the radiative losses until the reduced convection can balance the losses. Obviously a sizable temperature difference is needed, which implies also a compression and a sizable increase in the magnetic intensity up to the typical sunspot values. In this sense it is possible to say that sunspots are not brought up from below but are formed at the top of the convection zone by the supergranular motion which can produce local detours in the flow of energy. Sunspots seem to be secondary phenomena which appear if the necessary unconcentrated flux through the solar surface is provided in an active region by the buoyancy of a larger flux tube. But they do not form until so much flux is concentrated at one point that the cooling mechanism can overcome the radiative flux from the neighboured subphotosphere.

Before I leave the subject of sunspots I should say a few words about their lifetime. For this lifetime we have three options:

First the Cowling timescale, which is very long and therefore should be ruled out. I dare to make this statement despite the results of Kopecký and Kuklin (1966) and Schröter (1966), who have computed comparatively low timescales from the local conductivity in the umbra. I do not think that such a local timescale is applicable for the decay of the whole sunspot field. Even if the local currents in the umbra decay, the result would simply be a small bulge in the field at that level, but certainly all the flux deeper down or high up in the corona cannot contract towards that tiny ring, it will rather induce some additional currents in the high conductivity of its surroundings and then stop worrying about the conductivity at the umbra. But I should emphasize that nevertheless these low conductivities in the umbra and also in the photosphere are of importance for the whole solar cycle, because in just this level occurs the dissipation and reconnection of opposite fluxtubes, which meet stochastically at the borderlines of supergranules and granules. 
The next choice for a sunspot lifetime is the radiative relaxation time which has the right order of magnitude, as Weiss (1964b) has estimated. But an umbra does not seem to shrink smoothly, and the cooling mechanism which holds the spot stable will not stop unless sufficient flux has been transported away from the umbra.

The third possibility seems to be the timescale for destruction of the spot through the action of the supergranulation which may chip off the flux in small pieces, as Simon and Leighton (1964) have proposed. But this lifetime is hard to estimate, though the mechanism seems to be reasonably confirmed by observations.

\section{Alignment, Energy Storage, and Release}

We now have to deal with the active magnetic fields above the solar photosphere. Here the magnetic energy is predominant from the low chromosphere out into the solar wind for many solar radii. Since there is no serious competitor the magnetic field will be nearly 'force-free' in the sense that any sizable electrical currents which are able to change the magnetic field will flow along the field. But it would not be correct to say that the Lorentz force vanishes. This force is only small compared to what it could be if there would be comparable material energies present. The Lorentz force can be quite strong, however, in terms of the hydrostatic pressure gradient or gravity force. Therefore we will find many aligned structures in the solar atmosphere. The chromosphere is actually overcrowded with such aligned structures, as the excellent modern $\mathrm{H} \alpha$ filtergrams demonstrate.

In principle the force-free currents along the field can be deduced from the horizontal derivatives of measurements of the transverse field components, but such measurements do not often have sufficient accuracy. In many cases it seems to be more reliable to use the information available from fine-scale longitudinal magnetograms together with filtergrams. Especially for this purpose some years ago I described a method (Schmidt, 1964, 1965) to compute the full three-dimensional magnetic field from a longitudinal magnetogram under the fictitious assumption of vanishing currents. Such computed transverse fields can be directly compared with filtergrams. From the directional deviations one can infer electrical currents. The method has been used for aligned structures like prominences by Rust (1966), Hyder (1967), and Semel (1967), who on a large scale generally found good agreement between predicted field direction and aligned structure, which seems to imply that there are no large electrical currents of such scales. But it should be borne in mind that the deviations between predicted and observed directions are useful information about electrical currents. I should add one comment. We have not yet any observational proof of the nearly force-free nature of the field in the solar atmosphere. This is difficult to get because locally it can only be done if all three components are measured in a volume with sufficient accuracy that the data can be differentiated with respect to all three dimensions. Such a task seems to be almost hopeless and useless, 
too, because near the photospheric level of observation the field simply will not be force-free. A sensible test would therefore involve the integral structure over a large area, but that is difficult, too. Only if we are able to identify the two photospheric basepoints of an individual fieldline, we could test whether all the current leaving at one basepoint is arriving at the other end. Simple tests for the twisted nature of the field do not prove that the current is actually parallel to the field.

If the atmospheric fields are nearly force-free, what are the proper boundary conditions? Since on the fieldlines the ratio of current and field intensity stays constant, we have one real characteristic. The other two are imaginary as for current-free fields. We therefore have to prescribe the flux everywhere at the boundary and additionally the current either in that part of the boundary where the flux is positive or where it is negative. If we exclude closed flux tubes in the atmosphere, there will be only one solution. Unfortunately the real situation is much more complicated and the natural boundary conditions are quite different. Once a fieldline has crossed the surface it has two basepoints and these are independently transported by horizontal convection, but so that the relative topology of the fieldlines connecting the basepoints is preserved if we forget about the extremely slow dissipation processes in the high temperatures of chromosphere and corona. The somewhat faster dissipation in the photosphere merely causes a slow slip of the basepoints relative to the convective circulation and occasionally a reconnection by annihilation of two encountering basepoints of opposite polarity. If the field is to be force-free no further condition can be put on the direction of the fieldlines, at the boundary. These boundary conditions will leave enough freedom so that the solution is not determined unequivocally. There arises a stability problem which may be important for the flare problem, as Gold (1964) has pointed out. The convection will steadily put energy into the field by moving the basepoints around. Thereby it will induce currents along the field and work against Lorentz forces acting in the surface. This energy storage will be the source of the flare energy, as many would agree upon. Now there may be more than one solution to a given boundary condition and gradual change of the boundary conditions by convection may put the magnetic field into a metastable solution different from the one with the lowest energy. Then by a finite disturbance a sudden change into the latter solution may be triggered off, so that the energy difference between the two solutions is released without any dissipation of magnetic flux. The latter point is of importance, since the timescales for dissipation of flux are so long that Parker (1963b) argues against any flare mechanism involving such processes. In this context it is also important to note that a large part of the flare energy is released at the very beginning of the flare into non-thermal motions.

In Munich we have tried to find a simple model to prove the existence of such metastable force-free fields which after a finite disturbance can release energy within the timescale of an Alfvén wave, because there is a state of lower energy accessible. The type of solar convection which most readily transfers energy into force-free currents 
along the atmospheric fields, is probably found in the penumbral filaments, which might be convection rolls aligned by the field, as Danielson (1961) has argued. Flare activity starts with the formation of the penumbra, so it seems to be reasonable to investigate models which might simulate the flux tubes between penumbral convection rolls of opposite spots. Meyer (1965) has shown that for a force-free field of cylindrical symmetry bounded by a cylinder of finite length so that the basepoints of the field are frozen into the two plane disks at the ends of the cylinder, there exists a state of lower energy if the pitch angle of the original field is of order unity. This state of lower energy is of course asymmetric and has a counterhelix to the side. Since it is not force-free, the energy difference for a corresponding force-free solution is even larger. This looked very promising, but one has to prove also the stability of the original solution for infinitely small disturbances because otherwise the convection of the basepoints represented as a rotation of one of the limiting disks would never put the field into the symmetric solution to begin with. I guess everyone here would expect this solution to be stable in this sense for very small pitch angles. At least we did. But the opposite was proven recently by Anzer (1968). To his own surprise he was able to show that any force-free field of cylindrical symmetry is unstable for infinitely small disturbances of a helical symmetry where the helix runs with the pitch of the original field. This simply means that in real nature there are not any force-free fields of cylindrical symmetry. Now we are trying to find them for a rotational symmetric model which might be a more realistic approach to the flare problem anyhow. By the way, if we consider penumbral filaments to be convection rolls and if we consider a flare to be caused by the twist induced in the field by these rolls, then it might be worthwhile to look into the Evershed effect during a flare.

Let me add a few remarks on loop prominences and loop structures in general. These most beautiful structures pose very difficult and fundamental problems. With this remark I do not refer to the intricate radiative and mass balance which Jefferies and Orrall (1964) and Kleczek (1964a) have studied some years ago. There is another difficulty involved in these innocent-looking simple structures, which occur during the course of a flare event and which obviously must be aligned by the field. Bumba and Kleczek (1961) and Kleczek (1964b) have shown that these filaments do not show any significant change in diameter if one follows them from base to base. Bruzek (1964) has shown that they are an essential phenomenon in the course of many flare events, especially so because they are based in the flare filaments themselves which overlie a region of enhanced field intensity. How can these structures exist in an almost forcefree magnetic configuration? E.g. a closed force-free loop cannot exist because it contradicts the virial theorem, as Cowling (1965) has noted. If one assumes as an initial configuration a toroidal loop imbedded in poloidal fields the loop must expand indefinitely and with Alfvén velocity because it cannot be balanced without Lorentz forces, arising e.g. from pressure gradients. Of course at the base of a solar loop structure there are Lorentz forces available which just hold the base in place or move 
it slightly with the horizontal convection. But I do not see how this can prevent the loop itself from rapid expansion into the corona which should take place with Alfvén velocity. Since this does not happen the loop may be compressed by some non-magnetic energy. If we assume only 100 gauss for the loop this energy must have a density of about $400 \mathrm{dyn} / \mathrm{cm}^{2}$ or a total amount of about $10^{32} \mathrm{erg}$ in a reasonable volume surrounding the loops. This seems to be a bit large even for flare conditions. If it were there it must expand also with high speed. So it would be extremely valuable to have reliable measurements of the toroidal magnetic field in a loop, because such measurements would inform us about the loop as well as about the post-flare conditions in the corona.

\section{References}

Anzer, U. (1968) Solar Phys., 3, 298.

Babcock, H.W. (1961) Astrophys. J., 133, 572.

Biermann, L. (1941) Vierteljahresbericht der Astron. Ges., 76, 194.

Beckers, J.M., Schröter, E.H. (1966) A.A.S. Meeting on Solar Physics, Boulder.

Bruzek, A. (1964) Astrophys. J., 140, 746.

Bullard, E.C. (1955) in Vistas Astron., Ed. by A. Beer, 1, 685.

Bumba, V. (1966) Varenna Summer School on Plasma Astrophysics.

Bumba, V., Howard, R. (1965) Astrophys. J., 141, 1492.

Bumba, V., Kleczek, J. (1961) Observatory, 81, 141.

Bumba, V., Howard, R., Smith, S. F. (1966) Proc. of the Meeting on Solar Magnetic Fields, Rome, p. 203.

Cowling, T.G. (1946) Mon. Not. R. astr. Soc., 106, 218.

Cowling, T.G. (1965) in Stellar Structure, Chicago, Vol. VIII, Chapter 8.

Cowling, T.G. (1966) Proc. IAU Symp. No. 22, North-Holland Publ. Co., Amsterdam, p. 405.

Danielson, R.E. (1961) Astrophys. J., 134, 289.

Danielson, R.E. (1965) Proc. IAU Symp. No. 22, p. 314.

Danielson, R.E. (1966) Proc. of the Meeting on Sunspots, Florence, p. 120.

De Jager, C. (1964) Bull. astr. Inst. Netherl., 17, 253.

Deinzer, W. (1965) Astrophys. J., 141, 548.

Elsasser, W. M. (1950) Rev. Mod. Phys., 22, 1.

Elsasser, W. M. (1956) Rev. Mod. Phys., 28, 135.

Gnevyshev, M.N. (1967) Solar Phys., 1, 107.

Godoli, G. (1966) Proc. of the Meeting on Solar Magnetic Fields, Rome, p. 289.

Gold, T. (1964) A.A.S.-NASA Symp. on the Physics of Solar Flares, p. 389.

Hale, G.E., Nicholson, S.B. (1938) Magnetic Observations of Sunspots 1917-1924 (Publ. Carnegie Inst. No. 498).

Hyder, Ch.L. (1967) Solar Phys., $2,49$.

Iroshnikov, R.S. (1965) Astr. Zu., 42, 494.

Jefferies, J.T., Orrall, F.Q. (1964) A.A.S.-NASA Symp. on the Physics of Solar Flares, p. 71.

Kippenhahn, R. (1963) Astrophys. J., 137, 667.

Kleczek, J. (1964a) A.A.S.-NASA Symp. on the Physics of Solar Flares, p. 77.

Kleczek, J. (1964b) Bull. astr. Inst. Csl., 15, 123.

Kopecký, M. (1966) Proc. of the Meeting on Solar Magnetic Fields, Rome, p. 285.

Kopecký, M., Kuklin, G.V. (1966) Bull. astr. Inst. Csl., 18 (in press).

Krause, F. (1965) Proc. IAU Symp. No. 22, p. 426.

Kulsrud, R.M. (1955) Astrophys. J., 121, 461.

Leighton, R.B. (1964) Astrophys. J., 140,1547.

Livingston, W.C. (1967) Science Journal, 3, 46. 
Livshitz, M.A. (1965) Solnezn. Aktibn., 2, 103.

Lortz, D. (1967) Physics of Fluids.

Meyer, F. (1965) private communication.

Parker, E.N. (1955) Astrophys. J., 122, 293.

Parker, E.N. (1963a) Astrophys. J., 138, 552.

Parker, E.N. (1963b) Astrophys. J., Suppl. 8, 177.

Parker, E.N. (1964) Astrophys. J., 140, 1170.

Pikelner, S. B. (1960) $\quad$ Astron. Zu., 37, 616.

Plaskett, H.H. (1959) $\quad$ Mon. Not. R. astr. Soc., 119, 197.

Plaskett, H.H. (1962) Mon. Not. R. astr. Soc., 123, 541.

Rust, D.M. (1966) Thesis Boulder 1966.

Schmidt, H.U. (1964) A.A.S.-NASA Symp. on the Physics of Solar Flares, p. 107.

Schröter, E.H. (1966) Proc. of the Meeting on Sunspots, Florence, p. 190.

Semel, M. (1967) Ann. Astrophys., 30, 513.

Severny, A. B. (1965) Observatory, 85, 183.

Sheeley, N.R. (1966) Astrophys. J., 144, 723.

Sheeley, N.R. (1967) Solar Phys., 1, 171.

Simon, G., Leighton, R.B. (1964) Astrophys. J., 140, 1120.

Steenbeck, M., Krause, F. (1966) Z. Naturf., 21a, 1285.

Stepanov, V.E., Grigorev, V.M. (1966) Trudy Sib IZMIR.

Weiss, N.O. (1964a) Phil. Trans. A256, 99.

Weiss, N.O. (1964b) Mon. Not. R. astr. Soc., 128, 225.

Weiss, N.O. (1965) Observatory, 85, 37.

Weiss, N.O. (1966) Proc. of the Meeting on Solar Magnetic Fields, Rome, p. 299.

Yun, H.S. (1967) Astron. J., 72, 838.

Zwaan, C. (1965) Rech. astr. Obs. Utrecht, 7, Part 4.

Zwaan, C. (1967) Solar Phys., 1, 478.

\section{DISCUSSION}

De Jager: Why do you rule out a sunspot life time in accordance to the low-conductivity values found by Kopecký and Schröter?

Schmidt: I do not think that such a local timescale is applicable for the decay of the whole sunspot field. Even if the local currents in the umbra decay, the result would simply be a small bulge in the field at that level, but certainly all the flux deeper down or high up in the corona cannot contract towards that tiny ring, it will rather induce some additional currents in the high conductivity of its surroundings and then stop worrying about the conductivity at the umbra. But I should emphasize that nevertheless these low conductivities in the umbra and also in the photosphere are of importance for the whole solar cycle, because in just this level occurs the dissipation and reconnection of opposite flux tubes which met stochastically at the borderlines of supergranules and granules.

Davis: It seems to me to be worthwhile to extend your brief remark on the possible mechanisms that might drive the solar differential rotation. Recent discussions show that the magnetic field in the solar wind exerts a substantial decelerating torque across the photosphere. If this decreases the angular momentum of the entire Sun, no spectacular effects are produced; but if it acts only on the convection zone, the rotation of the surface will be essentially completely stopped in a fraction of the life of the Sun. Thus the rotation of the surface must be driven by friction or magnetic coupling with a presumably slightly, or perhaps substantially, more rapidly rotating interior. The rotation rate of the surface layers is determined by a balance between the exterior torques exerted by the magnetic field and the interior torques. Since at present there seems no way to determine how either set of torques varies with latitude and with the surface-angular velocity, there is no way to predict what the surface-angular velocity should be. Consequently, no exotic mechanism is needed to produce differential rotation; and, if exotic mechanisms are present, there is no reason at present to expect that they can not be dominated by the torques mentioned above. 
Sturrock: Since the solar wind must flow along magnetic-field lines, some field lines must be open. The boundary of two open flux tubes of opposite sense comprises a sheet pinch, at which the field will not be force-free.

Schmidt: I completely agree that there are exceptions to the general rule that the atmospheric fields are force-free. The most important examples are the current sheets in the quiescent prominences and these sheets are often underlying the sheets you were referring to, as one can see from the work of Dr. Newkirk's group. 